\section{Mercantilización de los servicios de salud para el desarrollo: el caso de Colombia}

\author{
Oscar Echeverri ${ }^{1}$
}

Forma de citar: Echeverri O. Mercantilización de los servicios de salud para el desarrollo: el caso de Colombia. Rev Panam Salud Publica. 2008;24(3):210-6.

Palabras clave: servicios de salud, reforma en atención de la salud, equidad en el acceso, mercantilización, Colombia.

\footnotetext{
Banco Mundial, Colombia. La correspondencia se debe dirigir a: Oscar Echeverri, Ave 4 A, Oeste No. 5 186, Cali Valle, Colombia. Teléfono: 5728920430 Fax: 572 8920435. Correo electrónico: oecheverry@ emcalinet.co
}

Desde hace unas dos décadas se impulsaron reformas en los sistemas de salud de América Latina y el Caribe, cuya característica central ha sido la de tratar los servicios de salud como una mercancía bajo diversos esquemas de financiamiento y entrega. Estas reformas constituyen parte del enfoque del desarrollo de la región. El propósito de este ensayo es mostrar que el manejo de los servicios de salud como una mercancía en una economía de mercado no favorece el mejoramiento de la salud y, por tanto, va en contravía de los esfuerzos para incrementar el desarrollo, pues existe una relación circular entre la salud y el desarrollo en la cual la primera condiciona el desarrollo y viceversa. En el caso de Colombia, al cual se hará referencia especial, el cambio en la forma de financiar y entregar los servicios de salud como una mercancía ha conducido a un mayor grado de exclusión e inequidad para la población y a un deterioro marcado de la salud pública.

\section{LOS SERVICIOS DE SALUD: ¿UNA MERCANCÍA?}

En raras ocasiones se analiza la relación circular entre la salud y el desarrollo para decidir la forma en que se suministran los servicios de salud a la población, de tal manera que contribuyan al desarrollo y al bienestar de la población. Aquí sólo se mencionarán algunos aspectos esenciales de dicha relación, mientras se analizan los cambios que en las últimas décadas han determinado un nuevo y nocivo paradigma en la producción y suministro de los servicios de salud.

Fue precisamente al final de las décadas de los setenta y los ochenta del siglo XX, cuando ocurrieron tres hechos trascendentales en el mundo que cambiaron el paradigma de los servicios de salud en muchos países de la región latinoamericana: la privatización de las empresas del Estado, la mercantilización de los servicios de salud y el fracaso del modelo soviético.

El cambio del rol del Estado (Welfare State) en el manejo de empresas públicas que suministraban bienes y servicios públicos ocurrió por primera vez en Inglaterra con el gobierno de Margaret Thatcher y cobró fuerza en todos los países capitalistas. El argumento central fue la ineficiencia del gobierno para suministrar bienes y servicios a la población (creciente inflación, fracaso de los programas socia- 
les, erosión de la responsabilidad individual). La solución fue reducir el tamaño del Estado y transferir al sector privado la producción y el suministro de algunos bienes y servicios, entre ellos los de salud. Los economistas propusieron cambios propios de la economía de mercado para reducir el tamaño de la empresa y aumentar la eficiencia: Los jornales y salarios del Estado fueron congelados, recortados, comprimidos, erosionados, reducidos, etc., y las nóminas se acortaron con la eliminación de listas paralelas, jubilaciones tempranas, despidos, degradaciones, transferencias, atrición, re-localización, etcétera. Por otra parte, mercantilizaron ${ }^{2}$ bienes y servicios públicos para permitir que el mercado generara la competencia y fijara los precios.

Los pocos que pensaron en aplicar los cambios de la economía institucional en vez de la economía de mercado para mejorar la eficiencia del Estado, no fueron escuchados. Por ejemplo, los cambios para reducir el clientelismo y la corrupción y para saber cómo flexibilizar la legislación y cómo reducir la asimetría de información para que el Estado fuera más eficiente, sólo se comenzaron a explorar a mediados de los noventa, cuando se hizo evidente que los cambios de la privatización iniciados en los ochenta no mostraban gran cosa. El péndulo volvía a oscilar con timidez debido a un hecho trascendental: el capitalismo, que ya reinaba solitario (el Muro de Berlín había caído), tuvo el tiempo suficiente para mirar su propio ombligo y comprobar que tenía grandes llagas. De éstas se hablará más adelante. Simultáneamente con los cambios en el rol del Estado, los economistas cambiaron el carácter de "bienes meritorios" o "cuasi-públicos" de los servicios de salud y los convirtieron en una mercancía, ¡como los zapatos o las papas! No cabe duda de que debe haber una diferencia entre el acceso económico a las papas y aquel correspondiente a los servicios de salud. ${ }^{3}$ Este cambio ocurrió fundamentalmente por la "crisis" del sistema de salud en algunos países capitalistas debido a los altos costos, la creciente inflación, la exigencia de grandes capitales para diseminar nuevas tecnologías en el cuidado individual de la salud, y la ineficiencia de los servicios públicos de salud. Había que echar mano de la economía de mercado que, con su mano invisible, podría cambiar de manera eficiente la oferta y la demanda de servicios personales de salud (SPS) mediante la competencia y el libre juego de los precios en los servicios, y permitir el lucro del gran capital inclusive a expensas de la población y de los

\footnotetext{
2 La acepción inglesa commodification significa asignar el carácter de "mercancía" a un bien o servicio.

3 Los bienes de consumo masivo cuyo acceso es difícil negar son bienes meritorios, como la salud y la educación, o son bienes públicos, como la defensa nacional y la justicia. Los bienes de consumo particular cuyo acceso es fácil negar son bienes privados, como las mercancías.
}

mismos profesionales de la salud. La equidad y la inclusión eran asuntos relativamente ajenos a los economistas impulsores de estos cambios y la epidemiología era innecesaria para tomar decisiones en materia de salud. Olvidaron que la política de salud no se puede basar en el mercado, porque existe un importante segmento de población pobre que no participa de él en igualdad de condiciones, lo que implica una violación al derecho a la vida y a la salud con consecuencias negativas para el bienestar de dicha población.

En el fondo, la motivación del cambio fue principalmente el interés corporativo en generar lucro con un tipo de servicios cuya demanda no cesa de crecer debido a cambios demográficos y epidemiológicos en una población con usuarios mejor informados, y que permitiría la explotación de un enorme mercado para nuevas tecnologías. Sus fabricantes promovieron y aprovecharon el espejismo de que estas tecnologías fueran vistas como una panacea por la gente, e inclusive por los profesionales de la salud. Ni ellos ni las corporaciones de servicios de salud quieren recordar que desde la época hipocrática se sabe que, para mantener la buena salud, son elementos clave el ejercicio y la dieta sana, tener un hábitat sano, y no beber alcohol o hacerlo con moderación; además, hoy en día es bien conocida la importancia de abandonar el hábito de fumar y controlar el estrés. Para aprovechar esta añeja sabiduría, el gobierno asigna recursos exiguos, y sólo recientemente comienza a ser atractiva para derivar lucro con la inversión privada.

La tecnología es, quizás, el elemento que cambió dramáticamente la práctica de la medicina y la entrega de servicios de salud: el médico de familia y el médico de consultorio han ido desapareciendo por la exigencia de grandes capitales para la innovación y el uso de la tecnología. Esto lo aprovechó la organización corporativa para apropiarse del "negocio" de los SPS. El médico perdió el dominio sobre su "negocio" privado de varios siglos, donde la tradición era que atendiera al enfermo de acuerdo con las necesidades y regulara los honorarios según la capacidad del paciente para pagar. Esta transacción dependía, en esencia, de la capacidad del médico para cobrar. Sin embargo, este último pasó a ser un empleado del gran capital, propietario de la organización corporativa de SPS. Además de desalojarlo de su "negocio", entendido como su fuente de ingresos (más que de lucro desmedido), el gran capital no sólo lo ingresó como empleado sino que condicionó su ingreso a la "venta" de su clientela a la organización corporativa (usualmente una cadena de hospitales o una compañía de seguros de salud).

Este traslado del "negocio" de la salud a los conglomerados económicos no ha logrado reducir 
los costos ni ha creado coberturas de servicios suficientes, ni mucho menos ha producido una mayor equidad en el acceso a los servicios o mejores niveles de salud. Así, en los Estados Unidos de América (EUA) varios estudios $(1,2)$ muestran que los servicios de salud están fragmentados y descoordinados, son ineficientes y costosos y producen gran desperdicio. Schuster, McGlynn y Brooks (3) encontraron que sólo la mitad de los pacientes reciben la atención recomendada y que $30 \%$ reciben una atención contraindicada. Jacobs afirmó (4) que esta situación ha despertado rabia y desconfianza en el público por los servicios que recibe, expresadas en críticas en los medios de comunicación y ataques políticos. Blendon y cols. (5) notificaron que sólo una de cada ocho personas en los EUA piensa que el sistema es bueno, y más de una de cada tres $(37 \%)$ consideran que "el sistema tiene tantas cosas malas que debe ser totalmente reformado", mientras que sólo $14 \%$ de los canadienses opinan lo mismo. O sea que en los EUA, paraíso del libre mercado, los intermediarios del negocio de la salud son altamente cuestionados por la misma sociedad.

La tendencia creciente a vender los servicios de salud como una mercancía, también ha resultado negativa en Canadá. Además de los hallazgos en los EUA, "la falsa promesa del mercado" ha sido analizada por investigadores del Instituto de Investigación Tommy Douglas (6): "Dicho sucintamente, la histeria acerca de la 'crisis' en el sistema de salud está excesivamente inflada, es explotadora y particularmente egoísta. . . La pretensión de que no podemos cubrir el gasto de Medicare y [. . . ] que el suministro privado con lucro conducirá a un sistema más accesible y menos costoso, es indudablemente falsa".

El Consejo Canadiense para el Desarrollo Social (7) afirma que la respuesta está en el fortalecimiento del modelo actual no lucrativo: "La pretensión de los que proponen el mercado privado [...] no resultará en una mayor eficiencia ni en costos reducidos. Y ciertamente no redundará en mayor salud para los canadienses". Los argumentos a favor de los servicios privados de salud ignoran en absoluto décadas de evidencia acerca de que los servicios de salud que se brindan con fines de lucro son significativamente más caros, menos eficientes, más difíciles de auditar, a menudo de menor calidad y mucho menos accesibles, especialmente a los más vulnerables. Inclusive — iquién lo creyera!-, el Banco Mundial (8) en su Informe Anual del año 2004 afirma que no sólo es cierto en teoría, sino que en la práctica ningún país ha logrado mejoras significativas en materia de mortalidad infantil y educación primaria sin la acción del gobierno. Además, declara que la participación del sector privado en la salud y la educación es problemática sobre todo cuando se trata de llegar a los pobres, "pues están repletos de fallas del mercado [...] y por eso el sector privado, dejado a merced de sus mecanismos, no alcanzará el nivel de salud y educación que desea la sociedad".

George Soros dijo algo acerca de la medicina que es perfectamente aplicable a los servicios de salud: "La medicina es demasiado importante para dejarla a merced de los valores del mercado". Por otra parte, la corporativización de los servicios de salud ha llevado al editor del Atlantic Monthly, Arnold Relman (9), a afirmar que: "Los servicios de salud se han comercializado como nunca antes y el profesionalismo en medicina parece darle paso al empresarismo". Una nefasta consecuencia de esta situación es que hay más preocupación por la empresa y sus costos, que por el paciente, lo cual ha disparado la cifra de errores, desaciertos y muertes en el manejo de los pacientes ambulatorios y hospitalizados y la selección adversa en el aseguramiento, como lo afirma Chassin (10).

Aunque algunos economistas no quieran considerar el cuidado de la salud como un bien meritorio, ciertamente no pueden ignorar que "la mano invisible" del mercado no puede conducir a que dicho cuidado se brinde equitativamente, pues las fallas del "mercado de la salud" lo impiden. Insistir en el libre mercado de la salud es obligar a que los médicos enfrenten la disyuntiva de escoger entre sus pacientes y el rendimiento económico de las "empresas de salud" donde trabajan; entre el diagnóstico y tratamiento necesarios y el margen de ganancia para la empresa. Cabe decir que estas disyuntivas terminan haciendo cínicos, en vez de clínicos, a algunos profesionales de la salud.

\section{EL CASO DE COLOMBIA}

El artículo 366 de la Constitución Política de Colombia de 1991 establece que: "El bienestar general y el mejoramiento de la calidad de vida de la población son finalidades sociales del Estado. Será objetivo fundamental de su actividad la solución de las necesidades insatisfechas de salud, de educación, de saneamiento ambiental y de agua potable". Es claro que la salud se considera, al menos, como un bien meritorio, pero según Álvarez (11) la Constitución no la establece como un derecho. La reforma a los servicios de salud introducida por la Ley 100 de 1993 consistió básicamente en reemplazar el modelo público de oferta subsidiada de servicios, por otro de seguro obligatorio de salud que compra los servicios a "negocios" llamados eufemísticamente empresas promotoras de salud (EPS), que en realidad no fomentan la salud sino que más bien administran seguros. Estos "negocios" venden 
afiliaciones obligatorias a quienes pueden pagarlas y que a cambio reciben un plan obligatorio de salud (POS) de las instituciones prestadoras de salud (IPS) contratadas por las EPS para sus afiliados. Las IPS son tanto públicas como privadas, aunque cada vez más pasan a ser propiedad de las EPS. El Estado paga la afiliación de los pobres al "negocio" para que reciban de las IPS un plan obligatorio de salud subsidiado (POSS), que sólo tiene $60 \%$ de los beneficios del POS.

El sistema colombiano tiene serias debilidades pues, como sistema, es un engranaje de piñones en que todos deben ser del mismo metal y trabajar con las mismas tensiones para evitar un mal funcionamiento. En el caso colombiano, el piñón de las EPS es de metal (con la fortaleza económica para dominar el mercado), el de las IPS es de corcho (intermediarios), y el del usuario es de caucho (tiene que aguantar y acomodarse a lo que los otros piñones hacen) (figura 1). El resultado es que, con el tiempo, el piñón de acero daña el de corcho, y el de caucho se desestabiliza. Así, los resultados del sistema no pueden ser buenos. Al respecto, puede consultarse una elaboración más detallada de este tema en el artículo "Ley 100: Quo Vadis?" (12). Por otro lado, la rectoría, la regulación y la financiación son débiles, confusas y poco efectivas, pues no estaban preparadas para la competencia ni para vender servicios: el Ministerio de Salud se debilitó tanto con el modelo, que desapareció al fusionarlo con el de Protección Social, y nadie se quejó; la avalancha de decretos, resoluciones y nuevas leyes sobre el sistema de salud prácticamente sepultó la poca fun- cionalidad que tenía; y la evasión y la elusión debilitaron más las finanzas públicas.

Después de 10 años, en 2003 el problema central del nuevo modelo es que las coberturas de aseguramiento logradas son apenas de $56 \%$, siendo que la meta era la cobertura universal (100\%) cuando el gasto en salud subió de 7\% del PIB en 1990 a 10\% en 2000. De los asegurados, 30\% son afiliaciones obligatorias de los que pueden pagar, y $26 \%$ son pobres que asegura el Estado, cuando el total de indigentes es de más de $20 \%$ y el de pobres de cerca de $40 \%$. En el sistema anterior a 1990, 58\% de la población tenía acceso a servicios de salud, distribuidos de la siguiente manera: $35 \%$ en los correspondientes al Estado y 23\% en los servicios de la seguridad social o de los seguros privados de prepago.

En dólares, el incremento del PIB en salud entre 1990 y 2000 equivaldría a 2600 millones de dólares estadounidenses, sin que haya habido aumento de la cobertura. En otras palabras, la ineficiencia es alarmante y la universalidad del sistema establecido por la Ley 100 es un fracaso hoy. Recientemente el gobierno se inventó el subsidio parcial (aseguramiento con un número limitado de prestaciones) para tratar de estirar el financiamiento y aumentar así la cobertura, a costa de limitar el acceso a ciertas prestaciones básicas para la población subsidiada.

Según varios estudios, el costo de la intermediación en Colombia ( $16 \%-24 \%)$, es similar al de los EUA, de más del doble que en Alemania $(7 \%)$ y por lo menos una vez y media más que en Holanda

FIGURA 1. El sistema de salud colombiano

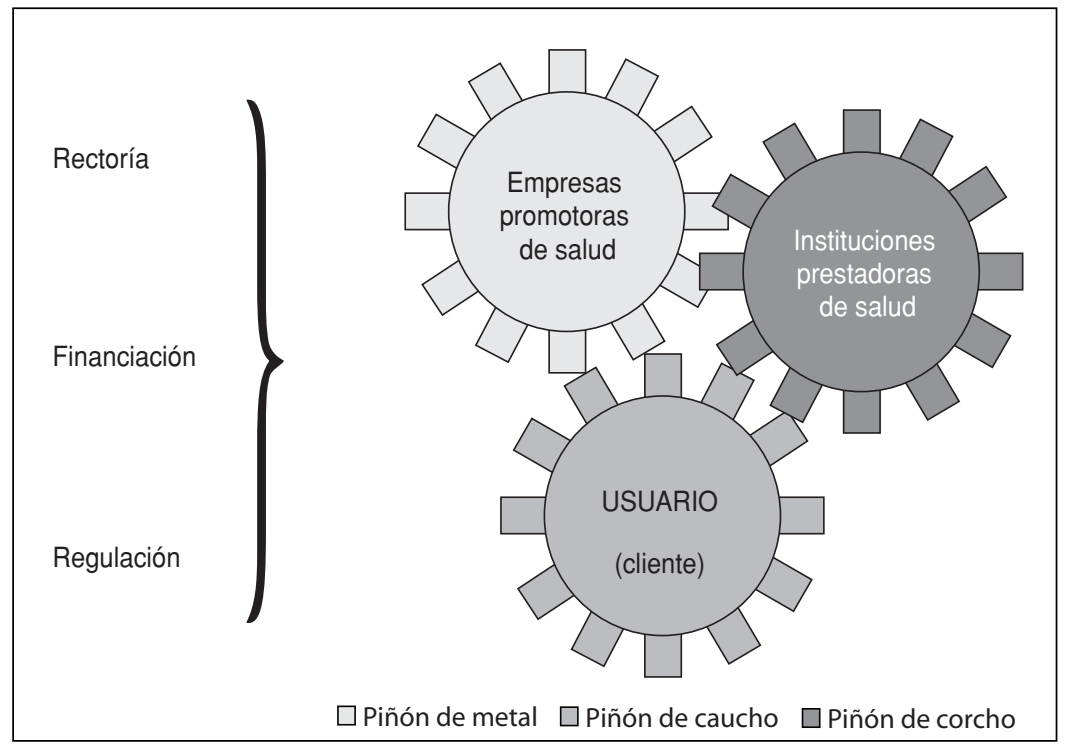

Fuente: elaboración propia. 
(9\%-11\%). Pero estar asegurado no es sinónimo de acceso y utilización de servicios. Muchos asegurados no usan los servicios y a veces se les niegan por razones burocráticas con que los del "negocio" tratan de eludir costos (selección adversa), y los proveedores (IPS) tratan de ahorrar costos (eficiencia), yendo en detrimento de la equidad, la calidad y la efectividad en muchos casos. Además, hay un claro sistema de primera y segunda clase: quienes compran seguros obligatorios tiene mejores servicios que los pobres que tienen afiliación por el Estado.

Las Empresas Sociales del Estado (ESE) que se meten al "negocio", están en desventaja competitiva con los "negocios" privados por múltiples razones: usan sus IPS, afectadas en muchos casos por el clientelismo, la falta de gestión y gerencia, el lastre de infraestructura inadecuada y subdotación de equipos, malos salarios, poco entusiasmo, falta de medicamentos y otros insumos, etcétera. Por otro lado, los mal llamados vinculados (no asegurados), que son una masa enorme de pobres e indigentes que difícilmente saben que el Estado debe asegurarlos, a menudo sólo utilizan los servicios públicos (en contadas ocasiones los privados) de urgencia, provocando una cartera para cobrar al Estado, que acostumbra ser moroso en sus pagos. Esto, unido a las ineficiencias administrativas de IPS públicas, ha hecho cerrar un número importante de hospitales en todo el país. Sin embargo, algunas encuestas muestran que de $80 \%$ a $85 \%$ de los afiliados al sistema están satisfechos con el servicio de salud, siendo mayor la proporción en los pobres. La pregunta que se hace: "¿Está usted contento con su servicio de salud?" es sesgada, ${ }^{4}$ pues es obvio que los pobres van a contestar que están satisfechos, independientemente del acceso que permite y la calidad de lo que compran, ya que es la primera vez en su vida que tienen un seguro. Velandia y cols. (13) encontraron "una serie de evidencias directas e indirectas de problemas relacionados con la calidad en la seguridad social (salud) que resultarían ser muy altas, más altas de lo que normalmente ha sido posible evidenciar".

El problema central sobre la Ley 100 es si es el esquema de la EPS como "negocio" —es decir, con fines de lucro- es el apropiado para garantizar el suministro de servicios de salud en Colombia. El debate se centra en la concepción de la salud como una mercancía cualquiera (bien privado), que se debe comprar obligatoriamente mediante un seguro, y que debe dejar un margen de ganancia suficiente para las EPS. ¡Es aberrante que haya que comprar una mercancía obligatoriamente, por ley!

En fechas recientes se aprobó una reforma a la Ley 100 (14) que trata parcialmente de subsanar fla-

\footnotetext{
4 Es lo mismo que si la abuela me preguntara: ¿usted quiere a su mamá?
}

grantes injusticias del sistema: la integración vertical de los intermediarios (EPS que construyeron sus propias IPS) se limitó con la contratación de no más de $30 \%$ en sus propias clínicas; se trató (después de 14 años de aplicar el modelo) de darle herramientas eficaces a la Superintendencia de Salud para hacer efectivas la inspección, vigilancia y control del sistema; se estableció la figura del Defensor del Usuario, y se buscó dar "progresivamente" los mismos beneficios del POS que tiene el régimen contributivo a los pobres que se inscriben en el inequitativo POSS. Además, como mea culpa ante el daño a la salud pública ocasionado por la Ley 100, la reforma propuesta incluyó la presentación obligatoria de un plan cuatrienal de salud pública. Razones de tiempo y espacio impiden un análisis de los posibles beneficios de esta nueva reforma.

\section{OTRAS EXPERIENCIAS EN AMÉRICA LATINA}

Aunque aquí no se trata de comparar las reformas ocurridas en América Latina, es importante señalar que Chile y Brasil produjeron cambios tan paradigmáticos como el de Colombia, con algunas particularidades que no han tenido resultados notablemente mejores a los de ese país.

Desde 1981, Chile fue pionero en establecer un modelo dual de servicios de salud que fija de entrada una discriminación en servicios de salud principalmente para los pobres (en general los más enfermos) a cargo del asegurador público Fondo Nacional de Salud (FONASA) y para los ricos (a menudo los más sanos) a cargo de las agencias aseguradoras privadas conocidas como Institutos de Salud Previsonal (ISAPRES). Titelman (15) afirma que esta estructura público-privada en un mercado altamente desregulado, ha contribuido a la discriminación de riesgos y al uso inapropiado de importantes subsidios cruzados. Varios cambios introducidos en los años noventa no han logrado corregir las deficiencias financieras ni las de acceso y equidad en los servicios de salud. La equidad no es uno de los objetivos del sistema de ISAPRES, pero sí lo es la eficiencia (contención de costos, incluyendo recortes en el cuidado necesario del paciente) para lograr su objetivo de lucro. Sin embargo, como afirma Hsiao (16), el libre juego de la oferta y la demanda no garantiza el aumento en la eficiencia debido a las fallas del mercado en salud (selección adversa, riesgo moral, discriminación de riesgos y asimetría de información). Aunque en teoría la población puede elegir el seguro público o el privado, en la realidad este último es el que elige a sus asegurados (los que pueden pagar) y los pobres "eligen" el seguro público. La solidaridad del sistema de salud 
chileno es mínima, pues como dice Nacuante (17), "no puede ser solidario un régimen en donde la protección de la salud depende primordialmente del monto de cotización que se entregue y que, en caso de la cesantía, la persona y su grupo familiar deban necesariamente dejar el sistema privado elegido y retornar al sistema público".

El "modelo universal" brasileño fue fruto del "movimiento sanitario" que comenzó en la década de los setenta y que culminó con la introducción de la salud como un derecho ciudadano y un deber del Estado, y la organización de un Sistema Único de Salud (SUS), público, universal e integral, en la reforma constitucional de 1998 (18). Su financiamiento sería mediante las rentas del Estado; así, este último garantizó la entrega de servicios a través del SUS con hospitales y centros de salud públicos (federales, estatales y locales) y la contratación de proveedores privados con y sin fines de lucro. La reforma se ha ido desvirtuando y hoy el SUS se ha dicotomizado al florecer un sistema de suplementación privada de los servicios públicos mediante planes voluntarios y planes prepagados de compañías de seguros. Según Fleury (19), la mayoría de los afiliados al sistema privado (30\%) utilizan los servicios públicos para emergencias, tratamientos complejos y enfermedades no cubiertas por los planes privados. De nuevo, el mercado estimuló la inequidad al estratificar a los usuarios según su capacidad de adquirir seguros privados y provocó estragos en la eficiencia y la equidad del SUS.

La realización de reformas en el sector salud se convirtió en una verdadera epidemia, pues con el paso del tiempo se demostró que la mercantilización de los servicios de salud es un error, de tal manera que se recurre a nuevas reformas que pretenden sanar los daños en la equidad, el acceso, la calidad y la eficiencia de los servicios de salud producidos por su mercantilización. Ugalde y Homedes (20) afirman que estas reformas, hechas con millonarias inversiones, no han logrado que los sistemas de salud en América Latina sean más equitativos, más eficientes o de mejor calidad. Además, han producido una creciente alienación en el recurso humano en salud.

\section{UNA REFLEXIÓN FINAL Y UNA PROPUESTA}

En los últimos dos siglos, la economía ha fracasado en resolver el problema de la pobreza en el mundo, pero ha tenido éxito en crear riqueza. Hoy son necesarias la filosofía y la ética para liderar la economía y así poder lograr un mundo con mayor cohesión social, con respeto por las diferencias, con el control de la codicia y la avaricia de los ricos, sin la envidia de los pobres, sin la arrogancia de los po- derosos y con la justicia social de una civilización menos egoísta y más ética. Esta reflexión importa cuando se trata de garantizar servicios de salud a la población, pues éstos deben contribuir a reducir la pobreza y la exclusión, no a aumentarlas.

Lo más patético de la mercantilización de los servicios de salud es el olvido de algunos economistas de las relaciones entre la salud y el desarrollo, al reducirlos exclusivamente al negocio de los servicios de distinto tipo suministrados por los trabajadores de la salud. La pobreza, la inequidad, la exclusión y la cultura, que son los determinantes más importantes de la salud y la enfermedad, se han soslayado al imponer modelos mercantilistas de servicios de salud. Esto no debería ocurrir, pues donde hay más pobreza hay menos salud. La observación repetida durante muchos años de trabajo en diferentes países de la región ha llevado al autor de este trabajo a concluir que los pobres permanecen pobres porque son pobres y están mal nutridos. Muchos de los pobres que no se desnutren pueden salir de la pobreza inclusive a pesar de la exclusión social. Por eso, el Estado tiene un gran papel y una enorme responsabilidad en proteger, mantener y recuperar la salud de toda la población con acciones intersectoriales (nutrición, vivienda, educación, esparcimiento, ambientes saludables, movilidad, cultura ciudadana, etc.) complementadas, no sustituidas, con los servicios personales de salud.

Hay un asomo de cambio en el mundo con relación a la visión de la pobreza. Desde 1989, sin un contrincante ideológico que lo mantuviera ocupado, el capitalismo pudo hacer un alto para reflexionar y constatar (miró sus llagas) que, por definición, es un sistema que genera desigualdad, que nunca ha sido equitativo y que tiende a excluir a los que están en desventaja. Esta reflexión ha conducido a un creciente interés por investigar sobre la exclusión, la inequidad, la cultura de la pobreza, considerados determinantes sociales de la enfermedad. Los europeos (21) ya lanzaron una propuesta para desarrollar una sociedad con cohesión social, equitativa, incluyente y respetuosa de la diferencia. ¿Podemos seguir el ejemplo en Colombia y en América Latina? No esperemos más para comenzar.

Los canadienses aseguran que fortalecer la red de servicios públicos gratuitos es vital para el bienestar de todos los ciudadanos. Por ejemplo, Yalnizyan y el Consejo Canadiense para el Desarrollo Social (22) afirman que la provisión de servicios gratuita crea una "mancomunidad social", donde los individuos desarrollan su talento y capacidad para participar en la sociedad. Al reducir el espacio entre los ciudadanos, fortalece la cohesión social. Los "bienes mancomunados" desmercantilizan la vida y permiten gozarla no como un privilegio sino 
como un derecho humano. La provisión de servicios de salud por parte del gobierno, en alianza con el sector voluntario, representa una alternativa mejor a la del sector privado y garantiza mayor responsabilidad pública. Colombia debería reconsiderar su sistema y cambiar la entrega de servicios de EPS con fines de lucro a organismos no gubernamentales que no buscan lucrar, bajo todos los argumentos aquí expuestos.

Una reflexión final: el problema no es el mercado, pues el mercado es neutro. El problema es cómo lo usan los mercaderes. Si lo que los mueve es la codicia, la avaricia, la envidia o el afán de lucro sin importar de dónde viene, existe un gravísimo problema social. Pero si al mercader lo mueve un interés de lucro justo y equitativo y el deseo de prosperar, él junto con los demás (cooperación), existe entonces una alternativa de solución razonable frente a los altos costos de los servicios de salud modernos. Las instituciones que mejor encarnan esta alternativa son los organismos no gubernamentales, que operan sin fines de lucro. En resumen, el imperativo de hoy no es económico: es ético.

\section{SYNOPSIS}

\section{Commodification of health care services for development: the case of Colombia}

This is a succinct analysis of the circular relationship between health and development and the changes occurring over recent decades regarding health care services production and delivery that have resulted in a new paradigm. From the late 1970s through the 1980s, three major, worldwide shifts occurred that changed health care services in Colombia and in other Latin American countries: the privatization of government entities, the commodification of health care services, and the failure of the Soviet model. Health care system reform in Colombia, considered by some experts to be a model, is an example of health care commodification that, 15 years later, has not achieved the coverage, nor the equity, nor the efficiency, nor the quality, that it should have. More so than the market, the problem has been with the market entities that seek disproportionate profits. A solution for this situation is to appeal to nonprofit organizations for the purchase and sale of health care services.

Key words: health services, health care reform, equity in access, commodification, Colombia

\section{REFERENCIAS}

1. Dill Ann E.P. Managing to care: care management and service system reform Hawthorn, N.Y.: Aldine Books Publishers; 2001.

2. Dranove D. The economic evolution of American health care: from Marcus Wellby to managed care. Princeton, N.J.: Princeton Paperbacks; 2002.

3. Schuster MA, McGlynn EA, Brooks RH How good is the quality of health care in the United States? Milbank Q. 2005; 83(4):843-95.

4. Jacobs AK. Rebuilding an enduring trust in medicine. A global mandate: Presidential Address American Heart Association Scientific Sessions. Circulation. 2005;111(25):3494-8.

5. Blendon RJ, Brodie M, Benson JM, Altman DE, Buhr T. Americans' views of health care costs, access and quality. Milbank Q. 2006;84(4):623-57.

6. Rachlis MR, Evans PL, Barer M. Revitalizing medicare: shared problems, public solutions. Vancouver: Tommy Douglas Research Institute; 2001.

7. Canadian Social Development Council. Equality, inclusion and the health of Canadians. Submission to the Commission on the Future of Health Care in Canada. November 15, 2001.
8. Banco Mundial. Informe sobre el Desarrollo Mundial 2004: Servicios para los pobres. México: Mundiprensa, Alfaomega Grupo Editor; 2004.

9. Relman A. What market values are doing to medicine. Atlantic Monthly 1992 March: 96-106.

10. Chassin MR. Is health care ready for Six Sigma Quality? Milbank Q. 1998;76(4): 565-91.

11. Álvarez Castaño LS. El derecho a la salud en Colombia: una propuesta para su fundamentación moral. Rev Panam Salud Publica. 2005;18(2):129-35.

12. Echeverri O. "Ley 100: Quo Vadis?" Colomb Med. 1996;27(1):39-41.

13. Velandia F, Ardón N, Cárdenas JM, Jara MI, Pérez N. Oportunidad, satisfacción y razones de no uso de los servicios de salud en Colombia, según la encuesta de calidad de vida del DANE. Colomb Med. 2001;32(1):4-9

14. Ley 1122 09/01/2007. Imprenta Nacional de Colombia. Diario Oficial 46.506.

15. Titelman D. Reformas al financiamiento del sistema de salud en Chile. Revista de la CEPAL 1999;69:184-93.

16. Hsiao WC. Abnormal economics in the health sector. Baxter Health Policy Rev. 1995;32(3):125-39.
17. Nancuante U. Sistema de salud chileno. Una propuesta histórica conceptual. En: FONASA. ¿Una reforma en marcha? Santiago de Chile: Fondo Nacional de Salud; Julio de 1999.

18. De Sousa CG. O SUS entre a tradição dos Sistemas Nacionais e o modo liberalprivado para organizar o cuidado à saúde. Ciênc. saúde coletiva 2007;.12(supl):1865-74.

19. Fleury S. Reformas de salud: ¿universal, dual o plural? Modelos y dilemas de atención en salud en América Latina: Chile, Brasil y Colombia. En:. Molina CG, Núñez del Arco J, eds. Servicios de salud en América Latina y Asia. Washington, D.C.: Banco Interamericano de Desarrollo; 2000. Pp. 10-29.

20. Ugalde A, Homedes N. Las reformas neoliberales del sector de la salud: déficit gerencial y alienación del recurso humano en América Latina. Rev Panam Salud Publica. 2005;17(3):202-9.

21. Council of Europe. Promoting the policy debate on social exclusion from a comparative perspective. Trends in social cohesion. Germany: Council of Europe; 2001.

22. Yalnizyan A. The growing gap: a report on growing inequality between the rich and poor in Canada. Toronto: Centre for Social Justice; 1998. 